\title{
CONSUMO DE SUSTANCIAS PSICOACTIVAS EN ESTUDIANTES UNIVERSITARIOS EN VILLAVICENCIO 2009-2010
}

\author{
PSYCHOACTIVE SUBSTANCES UNIVERSITY STUDENTS IN VILLAVICENCIO 2009-2010 \\ Mónica García-Baquero ${ }^{2}$, Luz Tobon-Borrero ${ }^{3}$, María Ramirez-Duarte ${ }^{4}$
}

\section{RESUMEN}

Introducción: El consumo de alcohol acompañado del consumo de tabaco es un fenómeno que se encuentra profundamente arraigado en muchas sociedades y se ha convertido en uno de los factores de mayor riesgo sanitario. Objetivo: Identificar la prevalencia del consumo de sustancias psicoactivas (alcohol y tabaco) en jóvenes de 14 a 20 años, estudiantes de primer semestre de las universidades que se encuentran en el municipio de Villavicencio. Materiales y Métodos: Estudio de tipo descriptivo exploratorio, de corte trasversal. La población está conformada por 1878 estudiantes; que para el segundo semestre del año 2009 cursaban el primer semestre de formación universitaria. Resultados: La prevalencia de consumo de alcohol en algún momento de la vida en la población universitaria de Villavicencio se sitúa en el 95.6\% (IC95: 93,9\%96,9\%; DE: 0.21). En relación con la prevalencia de consumo de cigarrillo en algún momento de la vida en la población universitaria de Villavicencio se halla en el 47.4\% (IC95: 43,9\%-51\%, DE: 0.49); el 34.5\% de ese consumo se inició entre los 14 y los 18 años. Discusión y Conclusiones: En general se encuentra que las prevalencias de consumo de alcohol en todos los casos están por encima de las cifras nacionales, en contraste con las de cigarrillo, las cuales se encuentran por debajo. El alcohol en los menores de edad es un asunto de gran relevancia en la población universitaria: Uno de cada cuatro (1/4) de los encuestados consumen alcohol y uno de cada diez (1/10) fuman. Aunque el alcohol y el cigarrillo son sustancias socialmente aceptadas, se encontró que la prevalencia de consumo de alcohol es superior. Ambas sustancias proporcionan al consumidor mayor liderazgo y ego entre sus grupo social, sin embargo es de resaltar que el alcohol inhibe los prejuicios sociales, es un facilitador de expresión emocional y potencializador de relaciones sociales, produciendo una serie de efectos o consecuencias que son interpretadas como positivas, lo cual puede ocasionar un mayor consumo. (Rev Cuid 2011; 2(2): 161-67)

Palabras clave: Prevalencia, Alcoholismo, Tabaquismo. (Fuente: DeCS BIREME).

\section{ABSTRACT}

Introduction: Alcohol consumption accompanied snuff is a phenomenon that is deeply ingrained in many societies and has become one of the greatest health risk factors. Objective: To identify the prevalence of psychoactive substances (alcohol and snuff) in children 14 to 20 years, first semester students of the universities that are in the city of Villavicencio. Materials and Methods: A descriptive, exploratory, cross-section. The population consists of 1878 students, that for the second half of 2009 completed the first semester of college education. Results: The prevalence of alcohol at some point in life in the university town of Villavicencio is located in the 95.6\% (IC95: 93.9\% -96.9\%, SD: 0.21). With regard to the prevalence of Cigarette smoking sometime in life in the university town of Villavicencio is located in $47.4 \%$ (IC95: $43.9 \%-51 \%$, SD: 0.49 ), $34.5 \%$ of this consumption was initiated between 14 and 18 years. Discussion and Conclusions: In general it is found that the prevalence of alcohol consumption in all cases are above the national figures, in contrast to the cigarette, which are below. Alcohol in the minors is a matter of great importance in the college population: One in four (one quarter) of respondents use alcohol and one in ten $(1 / 10)$ smoke. Although alcohol and smoking are socially acceptable substances, found that the prevalence of alcohol consumption is higher.

Key words: Prevalence, Alcoholism, Smoking. (Source: DeCS BIREME).

\section{${ }^{1}$ Articulo original de investigación}

${ }^{2}$ Especialista en Salud Mental. Magíster en Educación. (Pendiente sustentación tesis). Docente Ocasional Tiempo Completo Universidad de los Llanos.

${ }^{3}$ Magíster en Enfermería Materno Infantil, Docente Ocasional Tiempo Completo Universidad de los Llanos.

${ }^{4}$ Magister, Docente Ocasional Tiempo Completo Universidad de los Llanos.

Autor para Correspondencia: Prof. García-Baquero, Correo: mgbaquero@gmail.com celular: 3144742252.

Articulo recibido el 1 de Septiembre y aceptado para publicación el 13 de Octubre de 2011. 


\section{INTRODUCCIÓN}

Entre los riesgos de salud que causan la mayor morbilidad y mortalidad a nivel mundial se encuentran, sin lugar a dudas, el tabaquismo y el alcoholismo. El riesgo de consumo de alcohol y tabaco varía según la cultura, el país, el género, el grupo etario, las normas sociales; dicho consumo es elevado para las dos sustancias en la mayoría de los países de América Latina, donde su uso es promovido y considerado como una práctica aceptada en diversos aspectos de la socialización.

Frente al llamado internacional por aunar esfuerzos en esta situación mundial, Colombia, apenas se despertó hacia la década de los ochentas e inició con el interés por indagar acerca del consumo de psicoactivos: Pérez (1994) con el libro Sustancias Psicoactivas: Historia del Consumo en Colombia. Posteriormente en la década de los 90, el Ministerio de salud contrata el desarrollo de investigaciones sobre salud mental y consumo de psicoactivos en 1993 y 1996, y a la par, la Dirección Nacional de Estupefacientes lo hizo en 1992 y 1996.

Sin embargo, fue el Programa Estatal Rumbos el que incluyó como Política, investigar sobre el consumo de Sustancias psicoactivas (SPA) en Colombia, actualmente este organismo coordina el Observatorio Colombiano sobre Consumo de Sustancias Psicoactivas.

En el año 2008, el Gobierno Nacional de la República de Colombia, a través del Ministerio de la Protección Social (MPS) Y LA Dirección Nacional de Estupefacientes (DNE), con el apoyo de la Oficina de las Naciones Unidas contra la Droga y el Delito (UNODC), la Comisión Interamericana para el Control del Abuso de Drogas (CICAD) de la Organización de los Estados Americanos (O.E.A), y la Embajada de los Estados Unidos en Colombia. Se llevó a cabo el Estudio Nacional de Consumo de Sustancias Psicoactivas en Colombia, con el propósito de estimar la magnitud de este fenómeno, conocer sus características principales y establecer la percepción social del riesgo asociado a las diferentes sustancias de abuso, entre otros aspectos.

\section{Los principales resultados de este estudio son.}

Cerca del $45 \%$ de las personas encuestadas declaran haber consumido tabaco/cigarrillo alguna vez en la vida (56\% de los hombres y $34 \%$ de las mujeres). No obstante, sólo el $17 \%$ pueden considerarse consumidores actuales; es decir, han usado esta sustancia al menos una vez en los últimos 30 días (24\% de los hombres y $11 \%$ de las mujeres).Esta cifra equivale a 3,3 millones de fumadores en el país.

En cuanto al consumo de alcohol, $86 \%$ de los encuesta- dos declaran haber consumido alguna vez en la vida, y $35 \%$ dicen haber consumido en los últimos 30 días( lo que equivale a 6,9 millones de personas). Los hombres con un $46 \%$ manifiestan haber consumido alcohol en el último mes y entre las mujeres es el $25 \%$.

Siendo una problemática evidenciada a nivel mundial, no se encuentran investigaciones sobre el tema en el Departamento del Meta por tanto el programa de enfermería de la Universidad de los Llanos con apoyo de la Secretaria de salud Municipal puso en marcha una investigación con el objetivo de Identificar la prevalencia del consumo de sustancias psicoactivas (alcohol y tabaco) en jóvenes de 14 a 20 años, estudiantes de primer semestre de las universidades que se encuentran en el municipio de Villavicencio.

Esto permitió: Caracterizar el consumo de acuerdo con posibles patrones o tendencias generales en adolescentes, estimar la probabilidad acumulada de ocurrencia del primer uso de alcohol y tabaco y clasificar los datos obtenidos de tal manera que permitan organizar una base de datos real de la ocurrencia de este evento en la población de estudio en el municipio.

Los indicadores estadísticos sobre consumo (prevalencias de vida, de periodo y actual) obtenidos en este macro proyecto permiten dar una mirada real de la situación de consumo de tabaco y alcohol en los jóvenes universitarios de 14 a 20 años de edad del municipio de Villavicencio, y podrán ser el soporte para la presentación de planes y programas tanto en las instituciones universitarias como en las gubernamentales, encaminados a fortalecer la política de espacios libres de humo y favorecer la salud de la población escolarizada en educación media y universitaria del Departamento y del Municipio de Villavicencio.

\section{MATERIALES Y MÉTODOS}

Estudio de tipo descriptivo exploratorio, de corte trasversal. La población está conformada por 1878 estudiantes; que para el segundo semestre del año 2009 cursaban el primer semestre de formación universitaria, en 39 programas modalidad presencial en 2 universidades públicas y 6 universidades privadas ubicadas en el municipio de Villavicencio.

Esta investigación fue aprobada por el Instituto de Investigación de la Orinoquía Colombiana. (IIOC)de la Universidad de los Llanos, la participación de los estudiantes fue voluntaria y el consentimiento informado fue obtenido mediante diligenciamiento de un formato autoadministrado. La muestra (n) la constituyen 778 encuestados $41,4 \%$ de la población estudiada.El procesamiento de los datos se inicio a través de una base de 
datos corregida y validada bajo Excel 2007, análisis de frecuencias y distribución bajo EpiInfo2000, análisis bivariado y correlacional bajo SPSS licenciado por la Universidad de los Llanos y tablas de contingencia bajo Epidat 3.1.Variables del estudio. Tabaco: En su forma de cigarrillo. Alcohol etílico: En cualquiera de sus formas (cerveza, vino, licores destilados: aguardiente, ron, whisky, tequila, ginebra, brandy, etc.)Consumo: Uso de las sustancias lícitas anteriormente indicadas, una o más veces en un periodo de tiempo especifico, en particular los siguientes:

\section{RESULTADOS}

Tabla 1. Prevalencias de consumo de alcohol y tabaco/cigarrillo en universitarios de primer semestre. Villavicencio. Año 2009.

\begin{tabular}{|c|c|c|c|c|c|c|}
\hline UNIVERSIDAD & $\mathrm{N}$ & $\begin{array}{l}\text { OH alguna } \\
\text { vez }\end{array}$ & $\begin{array}{c}\text { Cig · } \\
\text { alguna vez }\end{array}$ & $\begin{array}{l}\text { OH en último } \\
\text { año }\end{array}$ & $\begin{array}{l}\text { Cig en ultimo } \\
\text { año }\end{array}$ & $\begin{array}{l}\text { Consumo último } \\
\text { mes }\end{array}$ \\
\hline UNILLANOS & $240(30.8 \%)$ & $\begin{array}{c}97.1 \% \\
\text { (IC95\%: 94.1-98.8) }\end{array}$ & $\begin{array}{c}29.2 \% \\
\text { (IC95\%:23.5- 5.4) }\end{array}$ & $\begin{array}{c}89.2 \% \\
(\mathrm{IC} 95 \%: 84.5-92.8)\end{array}$ & $\begin{array}{c}18.8 \% \\
(\mathrm{IC} 95 \%: 14-4.3)\end{array}$ & $\begin{array}{c}64.6 \% \\
(\text { IC95\%:58.2- } 0.6)\end{array}$ \\
\hline $\mathrm{UCC}$ & $219(28.1 \%)$ & $\begin{array}{c}94.5 \% \\
\text { (IC95\%:90.6-97.1) }\end{array}$ & $\begin{array}{c}53.4 \% \\
\text { (IC95\%:46.6-60.2) }\end{array}$ & $\begin{array}{c}93.6 \% \\
\text { (IC95\%:89.5-96.5) }\end{array}$ & $\begin{array}{c}39.3 \% \\
\text { (IC95\%:32.8-46.1) }\end{array}$ & $\begin{array}{c}79.5 \% \\
(\text { IC95\%:73.5-84.6) }\end{array}$ \\
\hline S. TOMAS & $114(14.7 \%)$ & $\begin{array}{c}98.2 \% \\
\text { (IC95\%:93.8-99.8) }\end{array}$ & $\begin{array}{c}44.7 \% \\
\text { (IC95\%:35.4- 54.3) }\end{array}$ & $\begin{array}{c}96.5 \% \\
\text { (IC95\%:91.3-99) }\end{array}$ & $\begin{array}{c}35.1 \% \\
\text { (IC95\%:26.4-44.6) }\end{array}$ & $\begin{array}{c}86 \% \\
(\mathrm{IC} 95 \%: 78.2-91.8)\end{array}$ \\
\hline UNIMETA & $112(14.4 \%)$ & $\begin{array}{c}98.2 \% \\
\text { (IC95\%:93.7-99.8) }\end{array}$ & $\begin{array}{c}79.5 \% \\
\text { (IC95\%:70.8-86.5) }\end{array}$ & $\begin{array}{c}97.3 \% \\
\text { (IC95\%:92.4-99.4) }\end{array}$ & $\begin{array}{c}66.8 \% \\
(\mathrm{IC} 95 \%: 59.3-77.2)\end{array}$ & $\begin{array}{c}95.5 \% \\
\text { (IC95\%:89.9-98.5) }\end{array}$ \\
\hline S. MARTIN & $30(3.9 \%)$ & $\begin{array}{c}80 \% \\
\text { (IC95\%:61.4-92.3) }\end{array}$ & $\begin{array}{c}43.3 \% \\
\text { (IC95\%:25.5-62.6) }\end{array}$ & $\begin{array}{c}80 \% \\
(\text { IC95\%:61.4-92.3) }\end{array}$ & $\begin{array}{c}43.3 \% \\
(\mathrm{IC} 95 \%: 25.5-2.6)\end{array}$ & $\begin{array}{c}86.7 \% \\
\text { (IC95\%:69.3-96.2) }\end{array}$ \\
\hline UNIMINUTO & $23(3 \%)$ & $\begin{array}{c}95.7 \% \\
\text { (IC95\%:78.1-99.9) }\end{array}$ & $\begin{array}{c}65.2 \% \\
\text { (IC95\%:42.7-83.6) }\end{array}$ & $\begin{array}{c}95.7 \% \\
\text { (IC95\%:78.1-99.9) }\end{array}$ & $\begin{array}{c}52.2 \% \\
(\mathrm{IC} 95 \%: 30.6-73.2)\end{array}$ & $\begin{array}{c}87 \% \\
\text { (IC95\%:66.4-97.2) }\end{array}$ \\
\hline ESAP & $20(2.6 \%)$ & $\begin{array}{c}90 \% \\
(\mathrm{IC} 95 \%: 68.3-98.8)\end{array}$ & $\begin{array}{c}30 \% \\
\text { (IC95\%:11.9-54.3) }\end{array}$ & $\begin{array}{c}75 \% \\
(\text { IC95\%:50.9-91.3) }\end{array}$ & $\begin{array}{c}20 \% \\
(\mathrm{IC} 95 \%: 5.7-43.7)\end{array}$ & $\begin{array}{c}60 \% \\
(\text { IC95\%:36.1-80.9) }\end{array}$ \\
\hline A. NARIÑO & $20(2.6 \%)$ & $\begin{array}{c}90 \% \\
(\text { IC95\%:68.3-98.8) }\end{array}$ & $\begin{array}{c}40 \% \\
(\text { IC95\%:19.1- 63.9) }\end{array}$ & $\begin{array}{c}90 \% \\
(\text { IC95\%:68.3-98.8) }\end{array}$ & $\begin{array}{c}40 \% \\
\text { (IC95\%:19.1-63.9) }\end{array}$ & $\begin{array}{c}75 \% \\
(\mathrm{IC} 95 \%: 50.9-91.3)\end{array}$ \\
\hline
\end{tabular}

Consumo en el último mes o consumo actual: La persona declara haber usado determinada sustancia una o más veces durante los últimos 30 días. Consumo en el último año o consumo por periodo: La persona declara haber usado determinada sustancia una o más veces durante los últimos 12 meses. Consumo alguna vez en la vida:

La persona declara que ha usado determinada sustancia una o más veces en cualquier periodo de su vida.

\section{Año 2009.}


La prevalencia de consumo de alcohol en algún momento de la vida en la población universitaria de Villavicencio se sitúa en el 95.6\% (IC95: 93,9\%-96,9\%; DE: 0.21 ); el $72.2 \%$ iniciaron el consumo entre los 16 y los 18 años. Mientras que la prevalencia de consumo de cigarrillo en algún momento de la vida en la población universitaria de Villavicencio se halla en el $47.4 \%$ (IC95: 43,9\%-51\%, DE: 0.49); el 34.5\% de ese consumo se inició entre los 14 y los 18 años.

Esta tendencia se mantiene con el consumo en el último año, pues la prevalencia de alcohol es de $92,2 \%$ (IC95\%: 90-93.9; DE: 0.26) y cigarrillo 36,6\% (IC95\%: 33.3-40.1; DE: 0.48).

El consumo global en los últimos treinta días es de $78 \%$ (IC95\%: 94.9-80.8; DE: 1.24), teniendo peso especifico mayor el consumo de alcohol con el 49\% (IC95\%: 45.452.2).

En general se encuentra que las prevalencias de consumo de alcohol en todos los casos están por encima de las cifras nacionales, en contraste con las de cigarrillo, las cuales se encuentran por debajo. No se encontraron diferencias significativas según el programa, aunque las prevalencias fueron ligeramente superiores en los estudiantes que cursan carreras semipresenciales.

Se observa una asociación significativa entre el consumo de cigarrillo y un patrón de consumo abusivo de alcoholen los menores de edad. Es posible que esta diferencia esté relacionada con factores sociales y culturales en este rango de edad.

Para explicar las asociaciones encontradas es importante tener presente que el consumo de cigarrillo es un patrón de conducta con determinantes genéticos y socioculturales. Como ejemplo puede anotarse que identificar mayor número de familiares fumadores en aquellos con más alta frecuencia de consumo de cigarrillo puede deberse a un factor genético que interactúa con un factor ambiental (imitación). En cuanto a la imitación, las investigaciones sugieren que la amistad se consolida en la medida en que se compartan características comunes, que generalmente preceden a la relación de amistad, situación que se evidencia en los jóvenes universtiarios.

La publicidad juega un papel crucial en el consumo de cigarrillo, pues está orientada principalmente a la población adolescente y aumenta el inicio y la persistencia del consumo.

Estos resultados sugieren que el consumo diario de cigarrillo se establece en la adolescencia temprana, lo que implica que el consumo de SPA lícitas no es una conducta pasajera en un número importante de adoles- centes. A su vez, el consumo de cigarrillo, junto con el de alcohol, son la puerta de entrada al consumo de sustancias ilegales, hecho notablemente relevante para tomar medidas internas incluyendo estrategias de promoción de estilo de vida saludable y prevención de uso y abuso de SPA lícitas en los programas de bienestar institucional.

La información recolectada permite ver que el consumo de SPA lícitas, especialmente de alcohol en los menores de edad es un asunto de gran relevancia en la población universitaria: Uno de cada cuatro (1/4) de los encuestados consumen alcohol y uno de cada diez (1/10) fuman. Siguiendo la tendencia nacional, las prevalencias de consumo de alcohol en las universidades representan proporciones más altas en comparación al cigarrillo.

Las cifras de consumo tienden a aumentaren el género femenino. En general se encuentra que las prevalencias de consumo de alcohol en todos los casos están por encima de las cifras nacionales, en contraste con las de cigarrillo, las cuales se encuentran por debajo. El análisis por sexo muestra que los hombres tienden a consumir ambas sustancias en mayor proporción que las mujeres, pero con un margen de diferencia estadística realmente estrecha. En algunos casos, sin que estadísticamente sea importante la diferencia, se observa que el consumo de las mujeres tiende a ser mayor que el de los hombres.

Sin embargo, las mujeres presentan mayor tendencia a mantener el hábito de consumo en ambas sustancias, principalmente en el de bebidas alcohólicas; las cifras de consumo de este género ya están igualando el de los hombres, refutando la idea popular que son ellos quienes más consumen: seis de cada diez (6/10) hombres, igual que seis de cada diez (6/10) mujeres ingieren bebidas alcohólicas; uno de cada diez (1/10) hombres y dos de cada 10 (2/10) mujeres fuman.

Teniendo en cuenta los efectos del consumo temprano y frecuente de alcohol, es imperativo darle un gran impulso al trabajo preventivo con niños (as), adolescentes, padres y comercios en los que se venda bebidas embriagantes. Igualmente intervenir el consumo de cigarrillo en los estudiantes universitarios principalmente en los de primer semestre, para mejorar la tendencia a iniciar el hábito en los otros semestres y así lograr un mayor impacto en los programas de prevención y promoción relacionados con el evento.Un factor de riesgo para el consumo de alcohol y tabaco es el fácil acceso que tienen los jóvenes a dichas sustancias, además la disponibilidad de la sustancia está a su vez, determinada por factores como el precio del producto en el mercado, las políticas públicas que regulan su venta y la publicidad en medios de comunicación, además el consumo de cigarrillos, según evidencias bibliográficas; sirve como 
puerta para el consumo de otras sustancias más problemáticas. Asimismo, los efectos cognitivos y fisiológicos de las sustancias deterioran el desempeño escolar, que sugiere una relación indirecta entre el consumo de cigarrillos y el rendimiento académico.Aunque el alcohol y el cigarrillo son sustancias socialmente aceptadas, se encontró que la prevalencia de consumo de alcohol es superior. Ambas sustancias proporcionan al consumidor mayor liderazgo y ego entre sus grupo social, sin embarga es de resaltar que el alcohol inhibe los prejuicios sociales, es un facilitador de expresión emocional y potencializador de relaciones sociales, produciendo una serie de efectos o consecuencias que son interpretadas como positivas, lo cual puede ocasionar un mayor consumo.

\section{DISCUSIÓN}

En general se encuentra que las prevalencias de consumo de alcohol en todos los casos están por encima de las cifras nacionales, en contraste con las de cigarrillo, las cuales se encuentran por debajo. No se encontraron diferencias significativas según el programa académico, aunque las prevalencias fueron ligeramente superiores en los estudiantes que cursan carreras semipresenciales.

Se observa una asociación significativa entre el consumo de cigarrillo y un patrón de consumo abusivo de alcohol en los menores de edad. Es posible que esta diferencia esté relacionada con factores sociales y culturales en este rango de edad. Las cifras obtenidas corresponden a los hallazgos en estudios similares. Comparándolas con las últimas nacionales los datos siguen con la tendencia del patrón de consumo de esta población con respecto al género y difiere en cuanto a la edad de inicio, la cual es mucho mayor en este estudio. Contrastando los datos con los nacionales de SPA ilícitas, el consumo de SPA lícitas, está por encima en el caso del alcohol, y por debajo en cuanto al consumo de cigarrillo (Situación de Salud en Colombia. Indicadores básicos 2008, MPS).

Los resultados indican correlaciones positivas entre el consumo de ambas sustancias, la edad y el género; más notablemente en el consumo de bebidas alcohólicas. El análisis por sexo muestra que en general los hombres tienden a consumir ambas sustancias en mayor proporción que las mujeres, pero que esa distancia no es muy grande. En algunos casos, sin que estadísticamente sea importante la diferencia, se observa que el consumo de las mujeres tiende a ser mayor que el de los hombres. Sin embargo, las cifras indican la tendencia del consumo en mujeres a igualar y aumentar el consumo de hombres.

Este comportamiento de mayor consumo en la población femenina ya se había estudiado por el marcado aumento de consumo de estupefacientes en los últimos años tanto en el ámbito nacional como internacional en este género. Como lo reconoce Alarcón Rosario y colaboradores (2) en una investigación sobre los factores que influyen en el consumo de estas SPA en las mujeres, la cual encontró que el factor de riesgo de mayor incidencia es la tolerancia a la frustración, seguido por el factor optimismo y los factores protectores control de Impulsos y empatía, factores que por lo tanto deben ser considerados para trabajarlos en las estudiantes de la universidad, dentro de los programas de las áreas de Desarrollo humano y de Salud de la división de Bienestar Institucional.Aunque el alcohol y el cigarrillo son sustancias socialmente aceptadas, se encontró que la prevalencia de consumo de alcohol es superior. Ambas sustancias proporcionan al consumidor mayor liderazgo y ego entre sus grupo social, sin embarga es de resaltar que el alcohol inhibe los prejuicios sociales, es un facilitador de expresión emocional y potencializador de relaciones sociales, produciendo una serie de efectos o consecuencias que son interpretadas como positivas, lo cual puede ocasionar un mayor consumo.

La variable de principal actividad se formula debido a que el hecho de tener una ocupación laboral puede influir en la imitación y construcción de hábitos de consumo en los jóvenes. Puede proteger del consumo al ser lo primordial según el encuestado y/o al usar el tiempo libre, o bien favorecerlo al propiciar espacios de esparcimiento por el horario, pares consumidores, solvencia económica y otros factores dependiendo del contexto.

Según los datos obtenidos hay cierta diferencia entre los que consumen y no dependiendo de su actividad principal, siendo mayor el consumo de ambas sustancias en los que estudian y trabajan. No se encontraron diferencias significativas según el programa académico.Para explicar las asociaciones encontradas es importante tener presente que el consumo de spa lícitas es un patrón de conducta con determinantes genéticos y socioculturales. Como ejemplo puede anotarse que identificar mayor número de familiares consumidores en aquellos con más alta frecuencia de consumo de cigarrillo o de alcohol puede deberse a un factor genético que interactúa con un factor ambiental (imitación). En cuanto a la imitación, las investigaciones sugieren que la amistad se consolida en la medida en que se compartan características comunes, que generalmente preceden a la relación de amistad, situación que se evidencia en los jóvenes universitarios.

\section{CONCLUSIONES}

La información recolectada permite ver que el consumo de Sustancias Psicoactivas lícitas, especialmente de alcohol en los menores de edad es un asunto de gran relevancia en la población universitaria: Uno de cada cuatro (1/4) de los encuestados consumen alcohol y uno 
de cada diez (1/10) fuman.

Siguiendo la tendencia nacional, las prevalencias de consumo de alcohol en las universidades representan proporciones más altas en comparación al cigarrillo. Las cifras de consumo tienden a aumentar en el género femenino.

En general se encuentra que las prevalencias de consumo de alcohol en todos los casos están por encima de las cifras nacionales, en contraste con las de cigarrillo, las cuales se encuentran por debajo.

Aunque el alcohol y el cigarrillo son sustancias socialmente aceptadas, se encontró que la prevalencia de consumo de alcohol es superior. Ambas sustancias proporcionan al consumidor mayor liderazgo y ego entre sus grupo social, sin embargo es de resaltar que el alcohol inhibe los prejuicios sociales, es un facilitador de expresión emocional y potencializador de relaciones sociales, produciendo una serie de efectos o consecuencias que son interpretadas como positivas, lo cual puede ocasionar un mayor consumo.El análisis por sexo muestra que los hombres tienden a consumir ambas sustancias en mayor proporción que las mujeres, pero con un margen de diferencia estadística realmente estrecha. En algunos casos, sin que estadísticamente sea importante la diferencia, se observa que el consumo de las mujeres tiende a ser mayor que el de los hombres. Sin embargo, las mujeres presentan mayor tendencia a mantener el hábito de consumo en ambas sustancias, principalmente en el de bebidas alcohólicas; las cifras de consumo de este género ya están igualando el de los hombres, refutando la idea popular que son ellos quienes más consumen: seis de cada diez (6/10) hombres, igual que seis de cada diez (6/10) mujeres ingieren bebidas alcohólicas; uno de cada diez (1/10) hombres y dos de cada $10(2 / 10)$ mujeres fuman. Teniendo en cuenta los efectos del consumo temprano y frecuente de alcohol, es imperativo darle un gran impulso al trabajo preventivo con niños (as), adolescentes, padres y comercios en los que se venda bebidas embriagantes. Igualmente intervenir el consumo de cigarrillo en los estudiantes universitarios principalmente en los de primer semestre, para mejorar la tendencia a iniciar el hábito en los otros semestres y así lograr un mayor impacto en los programas de prevención y promoción relacionados con el evento.

Un factor de riesgo para el consumo de alcohol y tabaco es el fácil acceso que tienen los jóvenes a dichas sustancias, además la disponibilidad de la sustancia está a su vez, determinada por factores como el precio del producto en el mercado, las políticas públicas que regulan su venta y la publicidad en medios de comunicación, además el consumo de cigarrillos, según evidencias bibliográficas; sirve como puerta para el consumo de otras sustancias más problemáticas. Asimismo, los efectos cognitivos y fisiológicos de las sustancias deterioran el desempeño escolar, que sugiere una relación indirecta entre el consumo de cigarrillos y el rendimiento académico.

\section{RECOMENDACIONES}

Todo proceso de investigación es un acercamiento más al conocimiento de la realidad y a la aceptación de la misma en esta experiencia obtenida se ve claramente uno de los problemas fundamentales de la población universitaria en Colombia. Lo cual permite hacer las siguientes recomendaciones:

Realizar posteriores estudios para determinar las tendencias que toman los estudiantes al transcurrir el tiempo. Es relevante utilizar los datos recolectados por el área de salud de la división de Bienestar Institucional Universitario tanto en estudiantes de primer semestre como en el resto de la comunidad universitaria, para comenzar a implementar un sistema de vigilancia del evento (monitoreo epidemiológico) con estas sustancias que son aceptadas socialmente, para luego abordar las demás Sustancias Psicoactivas como cocaína, marihuana, bazuco, anfetaminas y heroína; y de esta manera obtener un tamizaje de las condiciones de salud con la que ingresan los estudiantes, con su respectivo seguimiento a lo largo de la carrera para desarrollar de una manera óptima el programa de estilos de vida saludable en la universidad.

Crear un programa que refuerce la política nacional para la reducción del consumo de sustancias psicoactivas y su impacto con la reducción de la demanda que trabaje de la mano con el plan de estudios.Los programas de prevención deben ser constantes e innovadores puesto que deben responder a las necesidades que se generan con las diferentes épocas, ya que el incremento diario en el comercio de sustancias obliga a desarrollar estrategias efectivas para el control y disminución de este flagelo.El inicio y el mantenimiento del consumo de Sustancias Psicoactivas lícitas durante la edad escolar o la adolescencia temprana sugieren que las actividades preventivas deben empezar tempranamente. Este proceso debe involucrar a toda la comunidad universitaria, especialmente a los coordinadores de las áreas del sistema de Bienestar institucional Universitario, quienes con sus funciones a cargo, pueden mejorar la situación ejecutando acciones dentro de su competencia con mayor alcance e impacto.

Determinar desde la admisión del estudiante sus redes de apoyo, factores de riesgo y factores protectores para poder brindar apoyo por medio del bienestar institucional. De la misma manera, se necesita de la participación de los docentes y directores de programa en acciones de 
educación en salud que promuevan conductas saludables, y en la identificación de estados emocionales negativos, especialmente en estudiantes con rendimiento inferior al esperado para su capacidad intelectual. Estos estados emocionales pueden precipitar y favorecer el inicio y la consolidación de un patrón de consumo diario de Sustancias Psicoactivas lícita.

Igualmente, el equipo del área de la salud debe aprovechar todas las oportunidades para evaluar la posible condición de consumidor de todos los adolescentes que demandan servicios, desalentar el consumo y utilizar todas las estrategias efectivas de que se disponga para incentivar el abandono del hábito en los consumidores.

Las universidades deben realizar estudios dirigidos a conocer las expectativas de los jóvenes con respecto a lo que les gustaría saber acerca de adicciones, sus motivaciones para evitar consumir drogas lícitas, y estas mismas deben promover el uso del tiempo libre en actividades que permitan el crecimiento y desarrollo de los estudiantes. La universidades deben fomentar actividades saludables como el deporte, talleres, y charlas en las que según las preferencias del estudiante participe de ellas; haciendo parte de requisitos institucionales (horas a cumplir, según lo establezca la institución), con el fin de que haya buen uso del tiempo libre y brindándole poco a poco mayor responsabilidad e independencia en su accionar diario.

Restringir la difusión del tabaco y bebidas alcohólicas, especialmente utilizando jóvenes como modelos para dicha publicidad. En cambio promover sobre el peligro de ingerir alcohol.

Finalmente, para hacer viable este proceso es fundamental la adopción de las políticas de salud pública que hagan efectivas las medidas que protegen a los no fumadores (como el crear espacios y respetar las áreas cerradas como ambiente libre de humo de tabaco, y la adecuación de zonas para fumadores), la restricción de la publicidad en algunos lugares y horarios y el aumento de los impuestos a los cigarrillos o la prohibición de su venta. En caso del alcohol, propender por la vigilancia por parte de la autoridad competente de los sitios de expendio de bebidas embriagantes cercanos de las sedes de la universidad que no respeten las distancias mínimas contempladas en el Plan de ordenamiento Territorial municipal.Es necesario determinar la prevalencia de la dependencia a la nicotina y al alcohol en esta población y diseñar programas de prevención para aquellos adolescentes que no han iniciado el consumo, y de promoción del abandono en los que actualmente beben o fuman, con énfasis en quienes muestran consumo de ambas sustancias.

\section{REFERENCIAS BIBLIOGRÁFICAS}

1. Camacho I; Consumo de alcohol en universitarios: relación funcional con los factores socio demográficos, las expectativas y la ansiedad social, fecha de citación: 10-09-2008, disponible en: http://www.scielo.org.co/scielo. php?script=sci_arttext\&pid=s012391552005000100006\&lng=e\&nrm=iso.

2. González R; Creencias en fumadores pertenecientes a un programa de salud cardiovascular, fechadecitación:12-09-2008, disponible en: http://scielo.isciii.es/scielo.php?pid=s113212962004000200004\&script=sci_ arttext\&tlng=es.

3. Gómez, S. LC. Un panorama nacional de la salud y enfermedad mental en Colombia: INFORME PRELIMINAR. Estudio Nacional de Salud Mental, Ministerio de la Protección social, Colombia, 2003.

4. Martínez J, Amaya W, Campillo H, Rueda G, Campo A, Díaz L; Consumo de sustancias psicoactivas en adolescentes, Bucaramanga, Colombia, 1996-2004, fecha de citación: 10-09-2008,disponibleen:http://www.scielosp. org/scielo.php?script=sci_arttext\&pid=s012400642007000200006.

5. Martínez J; Amaya W; Campillo H; Consumo diario de cigarrillo en adolescentes estudiantes: factores psicosociales relacionados con el género; disponible en: http://www.scielo.br/pdf/rlae/v16n5/es_17.pdf.

6. Ministerio de la Protección Social. y Dirección Nacional de Estupefacientes. Informe final. Estudio Nacional de consumo de sustancias Psicoactivas en Colombia. Editora Guadalupe S.A. Bogotá D.C. junio de 2009.

7. Pinzón L, Tobón O; Prevalencia del consumo de cigarrillo y características de los estudiantes fumadores de la facultad de ciencias para la salud de la universidad de caldas, fecha de citación: 12-09-2008, disponible en http:// promocionsalud.ucaldas.edu.co/downloads/revista\%203-4_8.pdf.

8. Rumbos. Programa presidencial para afrontar el consumo de drogas. Algunos términos básicos - Sustancias psicoactivas. En: Pilas con las drogas. Segunda edición. Bogotá, Colombia, 2000: 13.

9. Salazar I, Arrivillaga M; El consumo de alcohol, tabaco y otras drogas, como parte del estilo de vida de jóvenes universitarios, fecha de citación: 12-09-2008, disponible en: http://www.humanas.unal.edu.co/publicaciones/ publicaseriadas/psicologia/numero13/articulos_13/9_consumo_alcohol.pdf.

10. Téllez, M.J. Luces y sombras de la drogadicción en Colombia. Unimedios No. 97. Universidad nacional de Colombia. 2005. 